\title{
Repercusiones del 11 de septiembre en las bibliotecas
}

\author{
Por Ana Reyes Pacios Lozano
}

\begin{abstract}
Resumen: Se exponen y analizan las reacciones y efectos más destacados que han tenido lugar en las bibliotecas tras los actos terroristas del 11 de septiembre. La literatura especializada y las asociaciones profesionales, americanas e internacionales, ponen de manifiesto que los hechos tendrán repercusiones en el acceso a la información y en la gestión de las bibliotecas.
\end{abstract}

Palabras clave: 11 septiembre, Terrorismo, Asociaciones profesionales de bibliotecas, Presupuesto, Recursos sobre terrorismo, Planes de desastre, Seguridad.

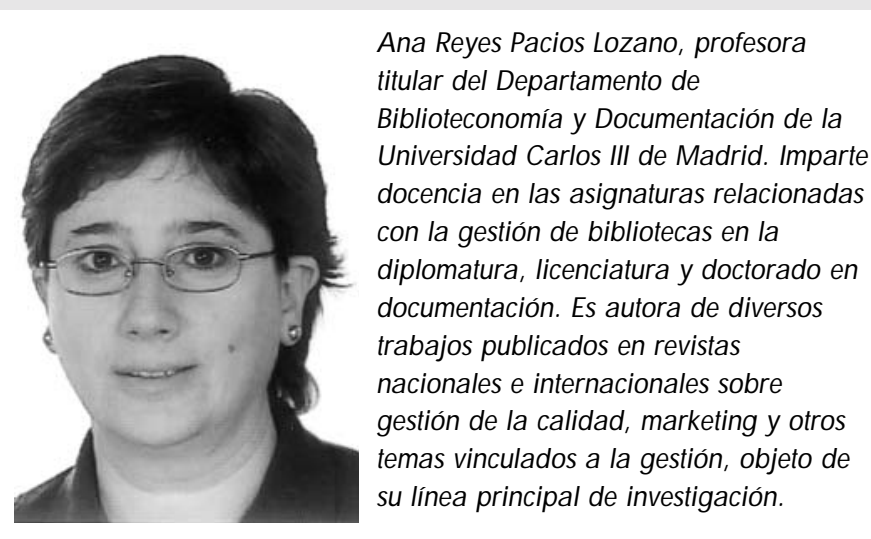

\section{Title: The aftermath of September $11^{\text {th }}$ in} libraries

\begin{abstract}
An exposition and analysis of the reactions of and effects on libraries after the terrorist attacks of September $11^{\text {th }}$ are presented. Both the professional literature and American and international professional associations affirm that the events and their aftermath will have repercussions affecting information access and library management.
\end{abstract}

Keywords: September 11, Terrorism, Professional library associations, Budgets, Resources on terrorism, Disaster planning, Security.

Pacios Lozano, Ana Reyes. "Repercusiones del 11 de septiembre en las bibliotecas". En: El profesional de la información, 2002, septiembre-octubre, v. 11, n. 5, pp. 361-369.

\section{Introducción}

Los actos terroristas perpetrados el 11 de septiembre de 2001 han tenido un impacto negativo en la economía, el consumo, la inversión, los viajes, el turismo, etc., y han creado trastornos en los mercados financieros así como repercusiones en todo el mundo, sin mencionar sus efectos en las relaciones internacionales y en la función de las organizaciones internacionales. De igual modo, también las bibliotecas e instituciones afines se han visto afectadas por estos hechos.

El objetivo de este trabajo es analizar, después de transcurrido poco más de medio año, cómo han vivido las bibliotecas americanas esta situación y qué impacto ha tenido en su entorno. Al instante de producirse el atentado ya se hizo patente la preocupación y reacción al mismo en muchas bibliotecas y organizaciones afines, no sólo del ámbito americano. Exponemos algunas de las respuestas más notorias. Algunas fechas dan una idea de la rápida reacción ante tales incidentes.

\section{Destrucción de bibliotecas, cierre de algunos servicios y medidas de control excepcionales}

El efecto más inmediato de los atentados fue la destrucción de 80 bibliotecas y la muerte de, al menos, 3 bibliotecarios especializados que trabajaban en compañías ubicadas en el World Trade Center'. Además, se vivieron grandes tensiones en las bibliotecas públicas del barrio de Manhattan los días siguientes a la tragedia. Aunque pretendieron seguir con su ritmo habitual, y de hecho 72 de las 85 sedes permanecieron abiertas, algunos servicios no se pudieron dar debido a la caída de la $\operatorname{Red}^{2}$. Según los bibliotecarios afectados, ya al día siguiente las bibliotecas intentaron responder a la situación con los recursos disponibles: dieron a la gente noticias e información de todo tipo basada en internet, ofrecieron la posibilidad de usar el correo electrónico e, incluso, refugio ante los ataques de terror ${ }^{3}$. Una idea del interés que se puso en ayudar a los ciudadanos es la respuesta de la Music Library Association cuyos miembros distribuyeron a través de la web una selec- 
ción de música clásica para ayudar a calmar los nervios de los más transtornados.

En Washington se cerraron varias bibliotecas ante incidentes relacionados con la localización de substancias sospechosas. El cierre de la Library of Congress a turistas y grupos es también un ejemplo significativo de medida preventiva tomada ante los posibles ataques de ántrax ${ }^{4}$.

En general, las bibliotecas públicas centrales de los estados donde se produjeron los ataques son las que están sufriendo más los efectos de la crisis debido a que son los edificios públicos más visitados. Se tiene muy en cuenta que los terroristas hicieron uso de internet y buscaron información desde algunos de estos centros $^{5}$. De ahí el temor que muestran sus bibliotecarios a posibles medidas restrictivas sobre el acceso a ellas y a la información, para prevenir su uso delictivo, reforzando o asegurando las condiciones de seguridad.

\section{Leyes y medidas antiterroristas estadouni-} denses que pueden interferir en la prestación de los servicios bibliotecarios

Al poco tiempo de producirse los ataques, la Administración de Bush empezó a emitir medidas antiterroristas $^{6}$. Entre ellas, algunas ligadas a la protección de los sistemas de información, como la Orden Ejecutiva 13.231 sobre protección de la infraestructura crítica en la era de la información, de 16 de octubre, cuyo fin es la protección de los sistemas de información frente al terrorismo cibernético? ${ }^{7}$.

\section{«Al instante de producirse el atentado ya se hizo patente la preocupación y reacción al mismo en muchas bibliotecas y organizaciones relacionadas con ellas»}

La ley Patriota contra el terrorismo (Provide appropiate tools required to intercept and obstruct terrorism act), firmada por el presidente el 26 de octubre de $2001^{8}$, establece la vigilancia de las comunicaciones como una de las herramientas esenciales para perseguir a los terroristas. Incluye disposiciones, vigentes hasta finales de 2005, concernientes a la intercepción del correo electrónico, internet y los teléfonos móviles. Bajo esta nueva ley se permite el espionaje de la Red usando nueva tecnología, conocida como Carnivore, que constantemente vigila el correo electrónico que circula. La policía podrá obtener los registros de llamadas, procedimiento denominado "trap and trace", cuyo resultado es la lista de todos los números de teléfono que han sido conectados desde una localidad en cuestión. A partir de ahora la vigilancia de internet re- cibirá este tratamiento incluyendo además, direcciones de correo-e, acceso a sitios web y las palabras que se eligen para hacer una búsqueda electrónica9. Faculta también a las autoridades encargadas de aplicar la ley a realizar cualquier orden de registro en todos los distritos y todos los estados de EUA, de forma que se facilitan las inspecciones en los domicilios de personas sospechosas.

Los cambios en la legislación norteamericana sobre cómo se comparte la información y la nueva legislación contra el terrorismo pretenden lograr un equilibrio entre las preocupaciones sobre la seguridad provocadas por los recientes ataques terroristas y la protección de las libertades civiles tradicionales. Sin embargo, todas estas medidas pueden representar una amenaza potencial a las libertades de todos los estadounidenses y atentar contra su privacidad. La ampliación de facultades que otorga la ley podría emplearse sobre otras muchas personas y no únicamente contra los sospechosos de terrorismo. De ahí que numerosos grupos hayan reaccionado manifestando su preocupación. Entre ellos, los profesionales de las bibliotecas para los que todas estas normas de prevención interfieren en el ejercicio de su trabajo y pueden llegar a suponer un reto a la profesión y a la ética profesional.

\section{Declaraciones y comunicados de instituciones y asociaciones relacionadas con las bibliotecas}

Ante los ataques, la situación de crisis desatada y las medidas establecidas por el gobierno de Bush, la respuesta de las asociaciones de bibliotecas no se hizo esperar. Las más importantes han emitido comunicados y resoluciones al respecto:

-Respuesta de la ALA (American Library Association) a la crisis nacional expresando sus condolencias a las familias afectadas y ofreciendo recursos informativos sobre el tema (12 de septiembre de 2001) ${ }^{10}$.

-Declaración firmada por la Aall (American Association of Law Libraries), ARL (Association of Research Libraries) y $A L A$, en la que manifiestan su preocupación ante propuestas legislativas que puedan ir en contra de los derechos de las personas y de la confidencialidad, elemento que consideran imprescindible tanto en la prestación de los servicios bibliotecarios como en el afianzamiento de la democracia ( 2 de octubre de 2001) $)^{11}$. Hacen objeciones a la colocación de aparatos destinados a capturar información del correo electrónico, la navegación por internet y de otros tipos de comunicación en la Red que permite conocer lo que el usuario ha visto e, incluso, la información personal remitida a una web. Por otra parte, consideran que se está expandiendo la definición de abuso y fraude informático de tal forma que algunos hechos de piratería 

informática podrían llegar a considerarse actividades terroristas.

-Declaración de la Ifla (Internacional Federation of Library Associations and Institutions) sobre Terrorismo, internet y acceso libre a la información (4 de octubre de 2001). Se defiende la libertad de expresión y el acceso libre a la información ante los argumentos que han utilizado algunas instituciones para restringir estos derechos y para incrementar la vigilancia. Aunque los terroristas hicieron uso de internet para preparar sus actos, también sirvió para responder a las necesidades de información en todo el mundo sobre los acontecimientos al instante de producirse y, especialmente, para que muchas personas pudieran verificar el estado y las condiciones de la gente afectada o de sus familiares. Estos razonamientos sirven, además, para considerar el acceso libre a la información como una estrategia para vencer al terrorismo que debe ser garantizada por cualquier profesional de la información y de las bibliotecas.

- Resolución de la $A L A$ reafirmando los principios de libertad intelectual a consecuencia de los ataques terroristas (23 de enero de 2002) ${ }^{12}$. Defienden algunos principios que podrían resultar afectados:

a. La difusión de la información y la defensa de la verdad necesarios en el ejercicio de los derechos de las personas.

b. Oposición ante la censura del gobierno a los medios de comunicación y a la supresión del acceso a la información gubernamental desclasificada.

c. Apoyo a la ética profesional para facilitar el acceso a la información sin monitorizarlo.

d. Animan a las bibliotecas y a sus trabajadores a proteger la privacidad y confidencialidad de la gente cuando usen la biblioteca, sus instalaciones y sus recursos.

e. Oposición al poder del gobierno para intimidar, suprimir, o coaccionar al hacer uso de la palabra.

-Comunicado del Imls (Institute of Museum and Library Services) titulado Bibliotecas y museos responden al ataque del 11 de septiembre en el que se apoya y destaca la labor de las bibliotecas y los museos como centros de recursos y de apoyo para la comunidad en la situación de crisis vivida tras los atenta$\operatorname{dos}^{13}$. Se indica que muchos ciudadanos acudieron a ellos buscando lugares de refugio seguros. Da la relación completa de los diferentes recursos y medios que han puesto a disposición de los ciudadanos para satisfacer las necesidades del momento. Otra medida llevada a cabo por esta institución fue la cancelación del National Museum and Library Service, un premio nacional anual concedido a bibliotecas y museos que ha- yan contribuido a que destaque de modo excepcional la comunidad a la que sirven, bien sea desde el punto de vista social, económico o ambiental ${ }^{14}$.

- También algunos representantes y cargos de asociaciones han expresado su visión de los hechos en editoriales de revistas. Así, Mary Reichel, presidenta de la American College and Research Libraries, hace hincapié en los diversos documentos que defienden la libertad intelectual, la libertad de expresión y la libertad de lectura ${ }^{15}$. Considera a esta última de gran relevancia en época de crisis, así como la libertad de comunicación, esencial para conseguir una sociedad libre y creativa. Promueve una filosofía de servicio y la igualdad en el acceso a la información sin discriminación alguna.

\section{«La planificación de desastres ha cobrado gran interés y los profesionales del sector sien- ten la urgencia de contar con un plan al respecto»}

Un artículo publicado en la revista American libraries recoge las frases de apoyo y adhesión a los bibliotecarios americanos por parte de otros colegas de diferentes países del mundo como Cuba, Georgia, Argelia, India, Argentina, Suiza y Gran Bretaña ${ }^{16}$.

-Oposición al uso de filtros de internet en las bibliotecas públicas de algunas ciudades como San Francisco y otras de California. La primera de ellas ha sido pionera en hacerlo desafiando la controvertida Childrens internet protection act (Cipa) a pesar de que puede costarle la pérdida de la subvención estatal. El software que filtra los contenidos ha sido condenado por las asociaciones defensoras de los derechos civiles ya que la prohibición de ver sitios que contengan ciertas palabras, como por ejemplo "sexo" o "pechos", limita el acceso a la información sobre la salud o las estadísticas por sexo. La Cipa ha sido cuestionada por importantes asociaciones, como el Electronic Privacy Information Center y la American Civil Liberties Union, que han interpuesto ante la justicia una demanda de inconstitucionalidad ${ }^{17}$.

\section{Sitios creados para satisfacer necesidades in- formativas sobre el terrorismo}

En ellos se puede encontrar cualquier tipo de información que tenga que ver con el tema. Su actualización es constante. Algunos ofrecen numerosos enlaces cuyos contenidos se podrían agrupar en los siguientes apartados ${ }^{18}: 1$. Artículos para comprender el terrorismo, el antiamericanismo y lo que ocurre en los países relacionados con él; 2 . Soluciones propuestas al problema del terrorismo; 3 . Prevención del terrorismo; 
4. El problema del terrorismo y los tipos de terrorismo; 5. Guerras ocasionadas por el terrorismo; 6. Racismo y discriminación; 7. Economía; 8. Energía; 9. Legislación; y 10. Recursos educativos.

Otro sitio de gran interés, creado por un grupo de bibliotecarios jurídicos, pretende recoger toda la legislación referida al tema ${ }^{19}$.

\section{Interés entre los profesionales por los temas de seguridad, prevención y preparación ante posi- bles desastres}

Una consecuencia clara después del 11 de septiembre es la lógica preocupación ante nuevos ataques terroristas. Al revisar la literatura especializada se pone de relieve la inquietud actual que existe en abordar temas relacionados con la seguridad ${ }^{20}$. Se insiste en prevenir este tipo de situaciones contando, por ejemplo, con un plan de desastre adecuado a cada tipo de biblioteca.

Varias asociaciones han respondido a esta demanda poniendo al día los recursos relacionados con los desastres. Así, la Public Library Association ha incluido en su página web (23 de septiembre de 2001), junto a la bibliografía y pautas para elaborar planes de desastre, un enlace en el que se dan indicaciones para actuar ante cualquier acto terrorista (bombas, explosiones, agentes químicos, agentes biológicos) ${ }^{21}$. Las recomendaciones están estructuradas en varios bloques:

- Lo que se considera necesario saber acerca del terrorismo antes de que ocurra algún acto de esta naturaleza. Por ejemplo, tipos de terrorismo y conocimiento de técnicas de preparación usadas en otras situaciones de crisis.

—Cómo actuar durante el ataque.

—Qué se debe hacer una vez ha tenido lugar.

También la $A L A$ ha actualizado su página correspondiente poniendo a disposición de los profesionales más recursos, bibliográficos y en texto completo, para bibliotecas de cualquier tamaño y tipo ante situaciones de emergencia ${ }^{22}$.

El énfasis en la prevención y la obsesión por la seguridad ha llevado a ciertos profesionales a entrar en el debate manifestando su opinión al respecto y haciendo propuestas según el tipo de biblioteca en la que ejercen su actividad. Así, Roy Tennant propone soluciones preventivas para las bibliotecas digitales ${ }^{23}$. Susan S. DiMattia recoge opiniones de varios responsables de bibliotecas especializadas relacionadas directamente con la tragedia del World Trade Center ${ }^{24}$. Otros piensan que es imposible estar preparados específicamente para actos terroristas como los sucedidos y manifiestan que lo más conveniente es estar prevenidos para perí- odos de emergencia ${ }^{25}$. Lo que se trasluce de todo esto es que la planificación de desastres ha cobrado gran interés y los profesionales del sector sienten la urgencia de contar con un plan al respecto.

\section{Reorganización y actualización de la página web de bibliotecas}

El objetivo es conseguir hacer frente a la situación y ayudar a entender los cambios que están sucediendo. Hay numerosos ejemplos de bibliotecas americanas, tanto universitarias como públicas, que han cambiado drásticamente su web. La lista de servicios, acceso al catálogo y otros enlaces habituales quedan relegados a un espacio pequeño para dar relevancia a lo que está ocurriendo y dar respuesta a las nuevas necesidades informativas de los usuarios. La totalidad de la página se llena con enlaces a recursos de información sobre terrorismo y los ataques a EUA. La imagen que trasmiten es de cercanía, de sentimiento con la ciudadanía, especialmente en las públicas. Se pone de manifiesto cómo colaboran ante la nueva realidad que se vive. Algunos de los títulos que agrupan estos recursos en las bibliotecas públicas son de sobra significativos: Recuperar, reconstruir, devolver: la huella del 11 de septiembre, en la biblioteca Newark de Nueva Cork; Comprender el cambio del mundo: recursos de información, en la Port Townsend de Washington; 11/9/01 Ataque y repercusiones: información, en la Clearwater de Florida, son algunos ejemplos.

\section{«El cierre de la Library of Con- gress a turistas y grupos es también un ejemplo significati- vo de medida preventiva toma- da ante los posibles ataques de ántrax»}

En los contenidos que forman parte de todos estos recursos informativos se nota una diferencia significativa entre las universitarias y las públicas: el carácter más práctico de la información que ofrecen las segundas. Una prueba de ello son las guías que proporcionan algunas bibliotecas puestas a disposición a partir de septiembre y octubre tanto en inglés como en español que indican, por ejemplo, el comportamiento antes, durante y después de un ataque terrorista, cómo elaborar un plan de desastre para la familia e incluso numerosas preguntas que se pueden hacer los ciudadanos. Es el caso de las precauciones que se deben tomar ante la amenaza del bioterrorismo, si se debe comprar una máscara antigás, tener una reserva de antibióticos en casa o beber agua del grifo, etc.

Los recursos están agrupados en bloques temáticos muy variados como, por ejemplo: Asesoramiento y 
asistencia a la familia; Asistencia legal; Búsqueda de personas desaparecidas; Servicios médicos; Empleo y negocios; Escuelas y colegios; Cómo ayudar mediante donaciones y voluntariado; Salud y seguridad; Terrorismo biológico y químico; Preparación ante los desastres; Legislación y seguros; Falacias, rumores, bromas y engaños; Noticias y medios de comunicación; Instituciones del gobierno; Recursos para padres y educadores, para niños, para inmigrantes; Recursos para favorecer el estudio del Islam, Afganistán y Oriente Medio, Terrorismo, Lecturas recomendadas, etc. ${ }^{26}$

En los centros universitarios se pueden encontrar fuentes más ajustadas al estudio del terrorismo y las organizaciones que lo amparan ${ }^{27}$. Para dar una idea del tratamiento del tema ponemos el ejemplo de la biblioteca de la Air University que ofrece 22 enlaces con fuentes de información sobre los grupos terroristas que operan en distintos países, entre los que se encuentra $E T A^{28}$. Otras fuentes aparecen agrupadas en enlaces cuyos títulos responden a temas tan diversos como: Leyes, Informes, Declaraciones del presidente, Recursos del gobierno, Afganistán, Ántrax, Bioterrorismo y terrorismo químico, Los árabes americanos, Respuesta internacional al terrorismo, La respuesta militar, Islam, Oriente Medio, Osama Bin Laden, Los talibán, Pasado y presente del terrorismo (doméstico e internacional), Datos sobre el 11 de septiembre, Víctimas del ataque del 11 de septiembre, Sitios educativos para ayudar a comprender a los niños lo que está sucediendo, Iniciativas de paz, Información sobre viajes, Líneas aéreas y alternativas al transporte aéreo, Donaciones y voluntariado, etc.

\section{Reducción del presupuesto destinado a las bi- bliotecas}

Antes del atentado la economía ya daba muestras de estar afectada, la inversión estaba decayendo y las bolsas ya habían empezado a deshincharse. Por eso parece obvio pensar que la situación se agravará más y la incertidumbre jugará un papel importante en una economía donde los medios de producción son de propiedad privada y cuyo funcionamiento está directamente determinado por las expectativas de beneficios. En consonancia, las previsiones en EUA son de una reducción de la producción económica, lo cual implica recesión. Se asoman así en el escenario recortes en los gastos sociales y en los impuestos para dejar más dinero en manos de contribuyentes y empresas, a fin de estimular tanto el consumo como la inversión y, por otra parte, ya han incrementado sus gastos militares. Todo afectará sin duda alguna a las bibliotecas en un futuro incierto y estamos asistiendo a las primeras reacciones en consecuencia.

Poco tiempo después de tener lugar los atentados ya se anunciaban recortes en el presupuesto para las bi- bliotecas y los profesionales empezaban a pensar en repriorizar sus gastos de acuerdo con esta nueva situación ${ }^{29}$. Hoy sabemos que la Administración de Bush ha disminuido en $100 \mathrm{M}$ US\$ el presupuesto correspondiente a dos programas de bibliotecas: educación y oportunidades tecnológicas. De ambos, hasta ahora, las bibliotecas recibían una pequeña cantidad y la $A L A$ los apoyaba. Además, el segundo proporcionaba algunos beneficios a entidades sin ánimo de lucro ${ }^{30}$. Sin duda alguna esto tendrá su reflejo, a su vez, en el acceso a la información entre los sectores de población más desfavorecidos si tenemos en cuenta, por ejemplo, los datos de uso de internet en la sociedad americana ${ }^{31}$. Según el estudio publicado en febrero de 2002, se incrementó un $25 \%$ anual entre las clases más pobres, en concreto un $33 \%$ y $30 \%$ entre la población negra e hispana. Estos dos colectivos forman parte del $10 \%$ de usuarios que se conecta desde las bibliotecas. Las restricciones presupuestarias en tecnología probablemente no van a contribuir a que aumente el número de personas que accedan a la Red a través de las bibliotecas públicas y, mucho menos, a que el $75 \%$ de los americanos pobres que no la usan lo hagan a partir de ahora.

\section{«Hay numerosos ejemplos de bibliotecas americanas, tanto universitarias como públicas, que han cambiado drástica- mente su web»}

Los 4,76 billones de US\$ de déficit en el presupuesto de Nueva York se notarán en gran medida en las instituciones culturales y los servicios sociales. El 1 de julio, al iniciarse el nuevo año fiscal, las bibliotecas de este estado habrán tenido que poner en marcha estrategias para poder hacer frente a los recortes presupuestarios anunciados. Supone un $15 \%$ menos, unos 39 M US\$ a repartir entre las instituciones culturales según el presupuesto presentado en el mes de febrero ${ }^{32}$. La primera respuesta no se ha hecho esperar y algunos servicios ya se han visto afectados: el cierre de algunos de ellos los domingos es una realidad. Esto tendrá, sin duda, repercusiones en la gestión de los recursos humanos de las bibliotecas.

La ejecución del nuevo presupuesto a lo largo de 2002 y 2003 nos permitirá conocer cómo se ha hecho frente a la crisis y las medidas que han adoptado los bibliotecarios para gestionar las contingencias de la mejor forma posible.

\section{¿Impacto en la comunidad bibliotecaria europea?}

Todo lo anteriormente anotado pertenece a la realidad de los EUA. Hasta el momento no se tiene noti- 


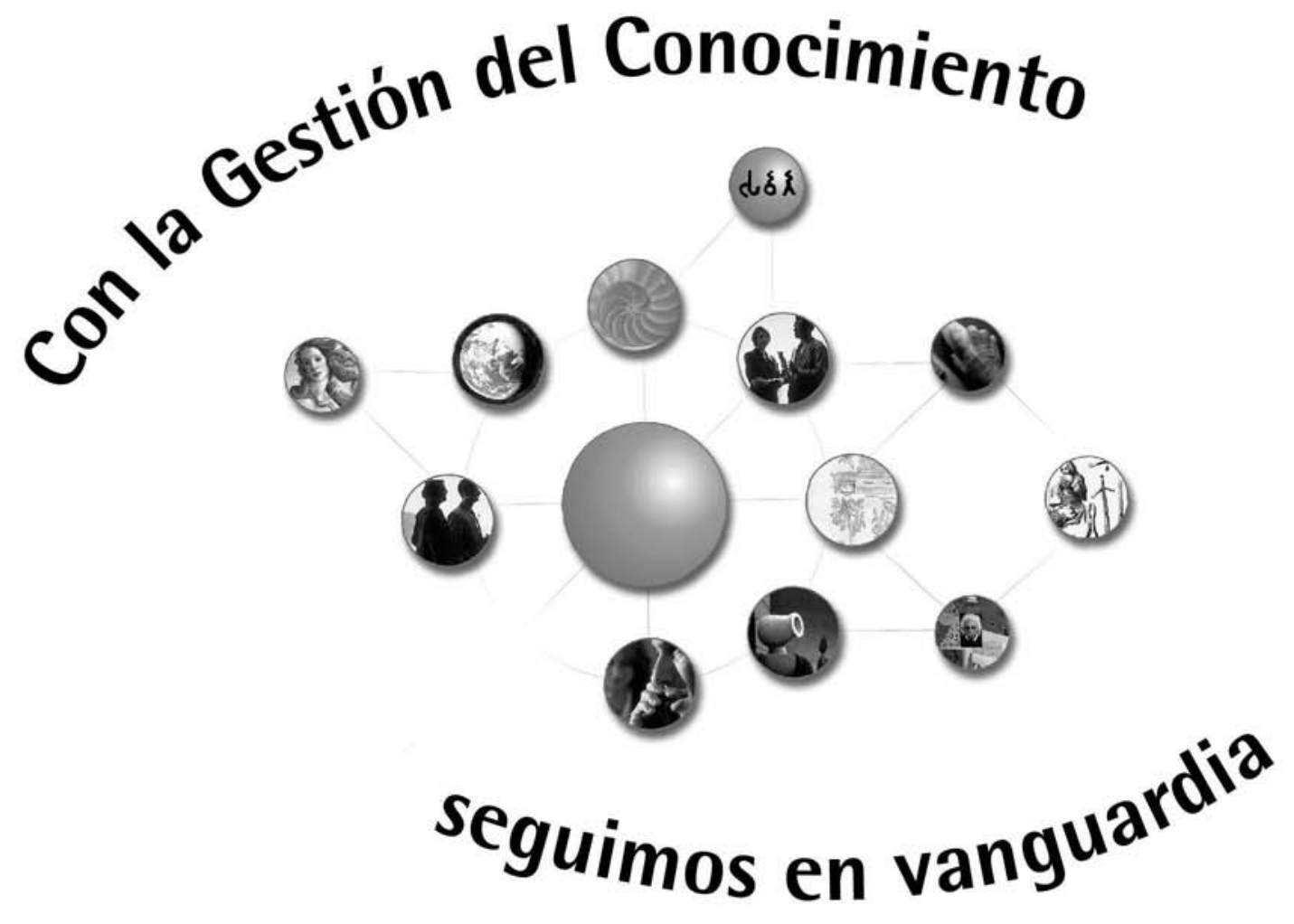

Estamos certificados en calidad ISO 9002

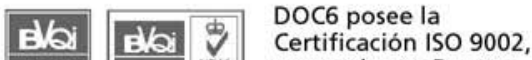
otorgada por Bureau Veritas Quality

International (BVQI), con las acreditaciones ENAC (Espeña) y UKAS (Reino Unido) para sus Centros de Barcelona y Madrid, dedicados a:

- Consultores en recursos de información

- Gestión del conocimiento

- Intranet/Extranet/Internet

- Edición electrónica

- Suscripciones a bases de datos y a revistas electrónicas (http://informame.doc6.es)

- Programas de gestión documental

- Cursos de formación
}

DOC6 S.A. ha recibido la etiqueta EUREKA de I+D para el proyecto COGNOS (n. 2734) de metodología para el desarrollo de mapas del conocimiento en las organizaciones.

Colaboramos con Vd. en encontrar las fuentes de información que precisa.

Visite el portal: http://informame.doc6.es A través del portal puede suscribirse a nuestro boletín de información gratuito sobre recursos electrónicos: info-NET

Con más de 14 años de experiencia, ofreciéndole nuestro servicio de calidad y con los mejores precios.

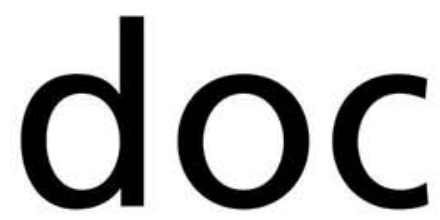

consultores en recursos de información

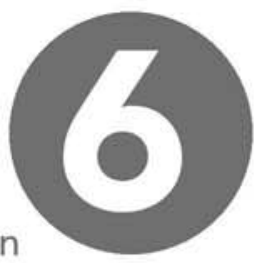

Mallorca, 272, planta 3 08037 Barcelona Tel. 932154313 Fax 934883621
Comandante Zorita, $8,2^{\circ} 4^{\mathrm{a}}$ mail@doc6.es 28020 Madrid

Tel. 915535207

Fax 915346112 
cia de situaciones parecidas en Europa y más concretamente en España. No obstante, teniendo en cuenta la habitual imitación de lo que se hace allí, podría llevar a que algunos países tomaran medidas similares en cuanto a leyes que extiendan el poder de las fuerzas policiales y supriman una parte sustancial de los derechos civiles. Más aún cuando, basándose en la Resolución de la ONU contra el terrorismo de 15 de noviembre de 2001, en la que se exhorta a los estados a intensificar su cooperación contra el terrorismo adoptando medidas en los terrenos económico-financiero y de intercambio de información y asistencia, vean justificada la emisión de tales medidas que afectarían seriamente a las bibliotecas y a sus profesionales. Probablemente, ante esta posibilidad, la Ifla se ha adelantado emitiendo el reciente Manifiesto sobre internet, aprobado por la Junta de Gobierno el 27 de marzo en La Haya y proclamado el 1 de mayo del $2002^{33}$. El texto se estructura en 3 apartados:

1. La defensa del libre acceso a la información como elemento imprescindible para la libertad, la igualdad, el entendimiento mundial y la paz.

2. Los principios de la libertad de acceso a la información a través de internet, en el que se destaca el derecho a la libertad de opinión y expresión, lo que incluye la libertad para tener opiniones sin interferencia y para buscar, recibir e impartir información e ideas mediante cualquier medio sin importar fronteras. Se hace hincapié en el respeto a la privacidad de los usuarios de las bibliotecas y en la confidencialidad de las fuentes de información y servicios que escojan.

3. La puesta en práctica del manifiesto, en donde se insta a la comunidad internacional y a los gobiernos a apoyar la libre circulación de información accesible a través de internet mediante las bibliotecas y servicios de información así como a oponerse a cualquier intento de censurar o restringir el acceso.

\section{«La Administración de Bush ha disminuido en 100 M US\$ el presupuesto correspondiente a dos programas relacionados con las bibliotecas»}

Después de esta reflexión, apuntando la posibilidad de reacciones en Europa con incidencia en las bibliotecas, sólo queremos anotar un dato curioso comentado en un foro de bibliotecarios de bibliotecas públicas madrileñas poco después de los hechos del 11 de septiembre que probablemente es común a muchas otras. La influencia de todo esto en sus bibliotecas se manifestó claramente en una alta demanda por parte de sus usuarios de libros sobre el Islam, los talibán y la figura de Bin Laden.

\section{Conclusión}

La situación después de los atentados afectará al uso de la información en las bibliotecas y al desempeño de los bibliotecarios en EUA. El impacto en su vida profesional ante las medidas legislativas establecidas se está dejando sentir. El bibliotecario se enfrenta a posibles retos en el ejercicio de su actividad relacionados con la defensa de los derechos de privacidad y confidencialidad de los usuarios de la biblioteca. Puede verse en situaciones críticas, como el reconocimiento de sujetos sospechosos en las que su ética se puede ver afectada ante la supuesta violación del derecho a la confidencialidad.

El clima de hostilidad que se vive en la sociedad americana hacia el mundo árabe en general tendrá también su impacto en los bibliotecarios americanos de origen árabe. La desconfianza puede perjudicarles en su actividad diaria. De hecho, ya se ha aludido al nuevo rol que deben desempeñar: facilitar la comprensión de la guerra de Afganistán, la situación de Oriente Medio y los registros en domicilios particulares para preservar las libertades civiles. Se les pide también que colaboren a la hora de proporcionar a los usuarios materiales objetivos para aprender sobre el Islam, los árabes, Oriente Medio y la religión musulmana ${ }^{34}$.

La respuesta eficaz de los bibliotecarios durante la tragedia y la ayuda prestada a los ciudadanos desde las bibliotecas ha potenciado su imagen. En consecuencia, probablemente, la sociedad americana les considerará una parte importante en el proceso de recuperación del país, cualquiera que sea su lugar de trabajo. Deberán contribuir en gran medida, con una gestión eficiente, a la viabilidad económica de su organización y, en definitiva, a la del país.

\section{Notas}

1. DiMattia, S. "Three WTC Libns., Remembered". En: Library journal, 2001, November, n. 1, p. 19.

2. Oder, N.; Kenney, B. "Attack on NYC, DC victimizes, strains libraries, librarians”. En: Library journal, 2001, October, n. 1, pp. 16-17.

3. Matthews, J.; Wiggins, R. W. "Libraries, the internet, and September 11". En: First Monday, 2001, December, n. 6, p. 12. Consultado en: 1804-02.

http://firstmonday,org/issues/issue6_12/matthews/index.html

4. Rogers, M.; Oder, N. "LC closes for antrax tests, scares around the country”. En: Library journal, 2001, November, n. 15, p. 14.

5. Kenney, B. J. "Central libraries in uncertain times". En: Library journal, 2001, November, n. 15, pp. 36-37.

$\mathrm{y}$

Oder, N. "Feds start to pull net information". En: Library journal, 2001, November, n. 15 , p. 16

6. Véase al respecto: Taylor, F. X. "Terrorismo: políticas y medidas antiterroristas estadounidenses". Consultado en: 18-04-02.

http://usinfo.state.gov/journals/itps/1101/ijps/pj63tayl.htm $\mathrm{y}$

Pillar, P. R. "Las herramientas antiterroristas". Consultado en: 18-04-02. http://usinfo.state.gov/journals/itps/1101/ijps/pj63pill.htm 
Desde enero del presente año Swets $\boldsymbol{\&}$ Zeitlinger Publishers, editora de esta revista, ha encargado la distribución de to das sus publicaciones a la siguiente empresa del grupo Swets $\boldsymbol{\&}$ Zeitlinger:

Turpin Distribution Centre. Blackhorse Road, Letchworth, SG6 1HN, Herts, Reino Unido.

Tel.: +44-146 267 2555; fax: 146248 0947

subscriptions@ turpinltd.com

Recordamos a nuestros suscriptores que continúan en funcionamiento los números de teléfono de atención al suscriptor en Barcelona:

Tel.: +34-932 701 144; fax: 932701145

7. Rodgers, P. "Proteger a Norteamérica del terrorismo cibernético". Consultado en: 18-04-02.

http://usinf.state.gov/journals/itps/1101/ijps/pj63fbi.htm

8. "Bush firma nueva legislación contra el terrorismo". Consultado en 18-04-02.

http://usinfo.state.gov/espanol/terror/01102607.htm

9. "Los derechos democráticos en E.E.U.U.: la primera víctima de la guerra antiterrorista de Bush". World socialist web site. Consultado en: 2702-02.

http://www.wsws.org/es/articles/2001/sep2001/span-s26.shtml

10. Consultado en: 04-04-02.

http://www.ala.org/pio/crisis/

11. Oder, $\mathbf{N}$. "Library groups find flaws in new antiterror legislation". En: Library journal, 2001, November, n. 1, p. 18.

12. Consultado en: 28-02-02.

http://www.ala.org/alaorg/oif/reaffirmifprinciples.html

13. Consultado en: 18-04-02.

http://www.imls.gov/pubs/sep11attack.htm

14. Oder, N. "Tragedy cancels Imls award”. En: Library journal, 2001, October, n. 1, pp. 17-18.

15. "September 11th and academic libraries". En: College \& research libraries, 2002, January, v. 63, n. 1, pp. 10-11.

16. Library Association. "Terrorist attacks shatter American sense of security”. En: American libraries, 2001, October, p. 21.

17. Castillo, C. "Voces contra la censura en los Estados Unidos". Puntonet, 2001, 13 de octubre, n. 51. Consultado en: 06-04-02.

http://puntonet.netfirms.com/es/esin1310.htm

18. Por ejemplo, el titulado biblioteca del terrorismo. Consultado en: 04 04-02.

http://www.terrorismlibrary.com/

19. Consultado en: 04-04-02.

http://l.staff.umkc.edu/lord//9-11-01-library/

20. Véase por ejemplo Bullard, S. W. "The dollars and sense of library security". En: Library administration \& management, 2001, fall, v. 15, n. 4, pp. 213-221.
21. Consultado en: 04-04-02.

http://www.pla.org/publications/technotes_disaster.html

22. Consultado en: 16-04-02.

http://www.ala.org/library/fact10.html

23. Tennant, Roy. "Coping with disasters". En: Library journal, 2001, November, n. 15 , pp. 26-27.

24. DiMattia, S. "Planning for continuity". En: Library journal, 2001, November, n. 15 , pp. 32-34.

25. Kenney, B. J. “Central libraries”, ob. cit, p. 37.

26. Anotamos algunos ejemplos de páginas web de bibliotecas públicas con recursos sobre terrorismo:

Consultado en: 04-04-02:

http://www.forsythlibrary.lib.il.us/news/

Consultado en: 21-02-02:

http://www.npl.org/Pages/InternetResources/SubjectGuides/emerg.html

Consultado en: 21-02-02:

http://www.brooklynpubliclibrary.org/eresources/wtc.htm

Consultado en: 21-02-02:

http://www.clearwater-fl.com/cpl/9-11-01events.html

Consultado en: 21-02-02:

http://wlo.statelib.lib.wa.us/ptpl/

Consultado en: 21-02-02:

http://www.kclibrary.org/resources/reference/wtc.cfm

Consultado en: 18-04-01:

http://www.saclibrary.org/InterResources/Sep11.html

27. Algunos ejemplos de bibliotecas universitarias con recursos sobre terrorismo son:

Consultado en: 05-03-02:

http://yaz.lib.indiana.edu/terrorism/

Consultado en: 22-01-02:

http://www.uab.edu/lister/bioterrorism.htm

Consultado en: 22-01-02:

http://www.jsu.edu/depart/library/graphic/usterror.htm

Consultado en: 22-01-02:

http://www2.lib.udel.edu/subj/godc/internet/terror/htm

Consultado en: 22-01-02:

http://www.library.vanderbilt.edu/romans/terrorism.html

Consultado en: 28-02-02:

http://www.library.ucla.edu/libraries/college/research/terrorism/

Consultado en: 28-02-02:

http://www.lib.rochester.edu/ref/tgovdocs.htm

Consultado en: 04-04-02:

http://ublib.buffalo.edu/libraries/units/lml/govdocsubj/terrorism.html

Consultado en: 04-04-02:

http://education.ed.pacificu.edu/newweb/Bailey/Trauma\&Children.html

Consultado en: 18-04-02:

http://www.wooster.edu/library/September11.html

28. Consultado en: 22-01-02:

http://www.au.af.mil/au/aul/bibs/tergps/tg98tc.htm

29. Oder, N. "Less money for PLs alter Sept. 11". En: Library journal, 2001, November, n. 1, p. 18.

30. Oder, N. "Benton: feds retreat from fighting the digital divide". En: Library journal, 2002, March, n. 15, p. 16.

31. "A nation online: how Americans are expanding their use of the internet". Consultado en: 18-04-02.

http://www.esa.doc.gov/508/esa.nationonline.htm

32. Oder, N. "NYC libraries face 15\% cut". En: Library journal, 2002, March, n. 15, p. 20.

33. Consultado en: 25-05-02

http://www.ifla.org/III/misc/im-s.htm

34. Chepesiuk, R. "Muslim-American librarians reflect". En: American libraries, 2002, January, pp. 40-42.

Ana Reyes Pacios Lozano, Departamento de Biblioteconomía y Documentación, Universidad Carlos III de Madrid. areyes@bib.uc3m.es 